\title{
Perioperative chemotherapy: the case for adjuvant chemotherapy for muscle-invasive bladder cancer
}

\author{
Alan So, MD, FRCSC
}

$\mathrm{D}$ espite aggressive surgical management, up to $50 \%$ of patients with muscle-invasive bladder cancer will have a tumour recurrence, which suggests that a significant proportion of these patients have metastases at the time of diagnosis. ${ }^{1}$ Hence, early application of multimodal therapy in bladder cancer is an attractive paradigm, especially to maximize outcomes in patients receiving aggressive local therapy by the immediate treatment of local and distant micrometastatic disease with chemotherapy.

\section{Neoadjuvant chemotherapy}

The benefits of using neoadjuvant chemotherapy in patients with muscle-invasive bladder cancer are unclear and as such should not be applied to all patients undergoing radical cystectomy. Most neoadjuvant chemotherapy randomized controlled trials (RCTs) have been underpowered, with significant methodological flaws that have resulted in uniformly poor results with minimal evidence of any improvement in survival. Although most of the neoadjuvant chemotherapy RCTs used cisplatin, drug protocols and combinations were heterogeneous among the trials. The only RCT to show a statistically significant benefit in overall survival was the Nordic 1 trial, ${ }^{2}$ which showed a benefit in survival only in cT3/T4a patients undergoing radiotherapy with neoadjuvant doxorubicin and cisplatin. The advantage of this neoadjuvant chemotherapy regimen was not found in patients undergoing radical cystectomy; this was addressed in the Nordic 2 trial. $^{3}$

The touted survival benefits of neoadjuvant chemotherapy are primarily derived from a compilation of 11 RCTs in the form of various metaanalyses, including the Advanced Bladder Cancer Meta-analysis Collaboration $(\mathrm{ABC})^{4}$ and the Cancer Care Ontario Meta-analysis. ${ }^{5}$ The ABC study, using data from 3005 patients, revealed that the relative benefit of chemotherapy is extremely modest, with a 95\% confidence interval $(\mathrm{Cl})$ for the hazard ratio (HR) that comes close to 1 , and an absolute survival improvement at 5 years of $5 \%$ (HR $0.86,95 \%$ Cl 0.77-0.95, $p=0.003) .{ }^{4}$

There are many issues with these meta-analyses that need to be addressed. All neoadjuvant chemotherapy meta-analyses included patients with heterogeneous chemotherapy regimens and, most importantly, none included the most popular combination used currently - cisplatin and gemcitabine. The importance of using optimal combination therapies is evident in the fact that in the $A B C$ analysis patients who received singleagent cisplatin actually had worse survival outcomes, compared with surgery alone. There is neither direct evidence for the use of cisplatin and gemcitabine in the neoadjuvant setting nor evidence to suggest the optimal number of cycles of chemotherapy.

Further, all meta-analyses addressing the benefit of neoadjuvant chemotherapy include studies with significant methodological flaws. The ABC study includes data from published and nonpublished trials questioning the quality of the data. ${ }^{6}$ The 2 large Nordic studies assessing cisplatin and adriamycin or methotrexate before cystectomy or radiotherapy included patients who had both neoadjuvant and adjuvant chemotherapy., ${ }^{3,7}$ Other deficiencies of the meta-analyses include the lack of definition of specific tumour or patient characteristics that were predictive of response and, more importantly, inclusion of trials in which most of the patients were younger, had excellent performance status and had good renal function. ${ }^{3,7-9}$ Hence, the efficacy and safety of neoadjuvant chemotherapy in patients with reduced performance status or other comorbidities is unknown and thus should not be a universally recommended treatment. 


\section{Overtreatment with neoadjuvant chemotherapy}

Using current standard chemotherapy regimens, neoadjuvant chemotherapy will always be associated with overtreatment; chemotherapy for metastatic bladder cancer produces only a $40 \%-60 \%$ response rate, implying many tumours are inherently chemoresistant and, accordingly, that a significant proportion of localized muscle-invasive tumours will not respond to chemotherapy.

This will obviously delay definitive local therapy in patients who are chemotherapy nonresponders. Using even the best imaging available, assessing tumour response may be difficult to ascertain; CT and MRI imaging is associated with up to a $42 \%$ discrepancy between clinical and pathological staging. ${ }^{10}$ The potential delay in chemotherapy nonresponders is a fundamental flaw in neoadjuvant chemotherapy since delays to radical cystectomy of greater than 12 weeks have been associated with poor outcomes. ${ }^{11-13}$ In summary, neoadjuvant chemotherapy has rather dubious clinical evidence and should not be recommended for all patients undergoing radical cystectomy.

\section{Advantages of adjuvant chemotherapy}

There are many potential advantages of giving chemotherapy in the adjuvant setting, compared with giving chemotherapy before surgery. The primary advantage is that local treatment is not delayed thus minimizing the risk of metastasis during the time from diagnosis to surgery. ${ }^{11-13}$ In addition, the concept of adjuvant chemotherapy allows the chemotherapy treatment to be tailored based on pathological criteria. The ability to risk stratify based on pathological stage is far superior to that of any other preoperative patient or tumour factors and it may prevent the overtreatment of good prognosis patients, such as those with $\leq$ pT2 disease who can expect up to an $80 \%$ 5-year recurrence-free survival. ${ }^{1,14}$ Thus adjuvant therapy allows for optimal timing of surgery and personalization of chemotherapy.

\section{Clinical evidence for adjuvant chemotherapy}

Similar to various neoadjuvant RCTs, several adjuvant RCTs have been undertaken (Table 1). However, unlike neoadjuvant trials, many of the adjuvant chemotherapy trials showed statistically significant benefits in survival. Skinner and colleagues ${ }^{15}$ randomized 91 patients to receive cisplatin, doxorubicin and cyclophosphamide versus observation after radical cystectomy. Patients receiving chemotherapy had superior time to progression and overall survival. Although criticized for methodological flaws, this study was the first to show the potential benefits of chemotherapy in an adjuvant setting.

Another well-known adjuvant chemotherapy trial is the German MVAC/ MVEC trial lead by Stockle. ${ }^{16-18}$ At 10 years, patients who received adjuvant chemotherapy had significant benefit in both cancer-free survival as well as overall survival; benefits were seen so early that the trial was stopped prematurely. In this study, most patients were highrisk ( $60 \%$ were $\mathrm{N}+$ and most were $\mathrm{pT} 4)$, pointing out the feasibility and potential benefits of tailoring chemotherapy to risk stratification based on

Table 1. Randomized controlled trials of adjuvant chemotherapy in patients with muscle invasive bladder cancer

\begin{tabular}{|c|c|c|c|c|}
\hline \multirow[b]{2}{*}{ Study } & \multirow[b]{2}{*}{$\begin{array}{c}\text { Chemotherapy } \\
\text { drugs }\end{array}$} & \multicolumn{2}{|c|}{ Group; no. of patients } & \multirow[b]{2}{*}{ Benefit } \\
\hline & & Chemotherapy & $\begin{array}{l}\text { No } \\
\text { chemotherapy }\end{array}$ & \\
\hline Skinner et al. ${ }^{15}$ & CAP & 47 & 44 & $\begin{array}{l}3 \text { yr DFS: } 70 \% \text { v. } 46 \% ; \\
\text { median survival: } 4.3 \text { yr v. } 2.4 \text { yr; } \\
p=0.006\end{array}$ \\
\hline Lehmann et al. ${ }^{18}$ & $M V(A / E) C$ & 26 & 23 & $\begin{array}{l}10 \text { yr DFS: } 41.7 \% \text { v. } 17.4 \% \\
10 \text { yr OS: } 41.7 \% \text { v. } 17.4 \%\end{array}$ \\
\hline Studer et al. ${ }^{21}$ & Cisplatin & 40 & 37 & No benefit; OS: $54 \%$ v. $57 \%$ \\
\hline Freiha et al. ${ }^{19}$ & CMV & 25 & 25 & $\begin{array}{l}\text { Benefit; DFS: } 50 \% \text { v. } 22 \% \\
p=0.01 \\
\text { OS: } 54 \% \text { v. } 34 \% \text { at } 5 \text { yr, NS }\end{array}$ \\
\hline
\end{tabular}


pathology. However, this study has been criticized, primarily for its small sample size, which was owing to its early discontinuation, as well as the fact that most patients with recurrences in the observation arm did not receive chemotherapy.

Freiha and colleagues ${ }^{19}$ compared patients with pT3 and pT4 bladder cancers treated with or without cisplatin, methotrexate and vinblastine. Like the Stockle study, the Freiha study was discontinued early owing to the benefit found at interim analysis of patients receiving adjuvant chemotherapy. Time to progression was longer in patients who received chemotherapy (37 v. $12 \mathrm{mo}, p=$ $0.01)$ at a median 62 months follow-up. The study was not powered to detect survival advantages.

A recent meta-analysis assessing adjuvant chemotherapy from 6 RCTs with a total of 491 patients showed that there was a $9 \%$ improvement in absolute survival at 3 years ( $\mathrm{HR} 0.75,95 \% \mathrm{Cl} 0.60-0.96, p=$ 0.019). ${ }^{20}$ However, the authors point out that the sample sizes for all of the studies were too underpowered and the results not "conclusive" enough to recommend adjuvant chemotherapy without more clinical evidence. In summary, although methodologically imperfect and not powered to determine overall survival differences, these RCTs show that adjuvant chemotherapy is feasible and safe, and, most importantly, that it may prolong cancer-free survival.

\section{Summary}

Based on all currently available clinical data, neoadjuvant chemotherapy is associated with significant overtreatment, flawed clinical trials as well as the lack of consensus of a chemotherapeutic protocol. Adjuvant therapy appears to be a more attractive concept, with many advantages and evidence of the benefit of time to cancer-free survival.

Assistant Professor, Department of Urologic Sciences, University of British Columbia, Vancouver, $\mathrm{BC}$

The positions provided in the Point/Counterpoint series are presented as general information and do not necessarily reflect the personal opinions of the authors.

This article has been peer reviewed.

Competing interests: None declared.

\section{References}

1. Stein JP, Skinner DG. Radical cystectomy for invasive bladder cancer: long-term results of a standard procedure. World J Urol 2006;24:296-304.

2. Malmstrom PU, Rintala E, Wahlquist $R$, et al. Five-year followup of a prospective trial of radical cystectomy and neoadjuvant chemotherapy: Nordic Cystectomy Trial I. The Nordic Cooperative Bladder Cancer Study Group. J Urol 1996;155:1903-6.

3. Sherif A, Rintala E, Mestad O, et al. Neoadjuvant cisplatin-methotrexate chemotherapy for invasive bladder cancer - Nordic cystectomy trial 2. Scand I Urol Nephrol 2002:36:419-25.

4. Neoadjuvant chemotherapy in invasive bladder cancer: update of a systematic review and meta-analysis of individual patient data Advanced Bladder Cancer (ABC) Metaanalysis Collaboration. Eur Urol 2005;48:202-5; discussion 205-6.

5. Winquist E, Kirchner TS, Segal R, et al; Genitourinary Cancer Disease Site Group, Cancer Care Ontario Program in Evidence-based Care Practice Guidelines Initiative. Neoadjuvant chemotherapy for transitional cell carcinoma of the bladder: a systematic review and meta-analysis. J Urol 2004;171:561-9.

6. Sternberg $\mathrm{CN}$. Perioperative chemotherapy in muscle-invasive bladder cancer to enhance survival and/or as a strategy for bladder preservation. Semin Oncol 2007:34:122-8.

7. Rintala E, Hannisdahl E, Fossa SD, et al. Neoadjuvant chemotherapy in bladder cancer: a randomized study. Nordic Cystectomy Trial I. Scand I Urol Nephrol 1993;27:355-62.

8. Nelli F. [The SWOG-8710/INT-0080 study: innovations and controversies concerning neoadjuvant treatment of locally advanced bladder cancer] [Article in Italian]. Suppl Tumori 2004;3:557-8.

9. Splinter TA, Jacqmin D, Roberts JT, et al. EORTC Group Study 30851: a phase II study of neoadjuvant chemotherapy in patients with invasive bladder cancer. Prog Clin Biol Res 1990;353:213-7.

10. Shariat SF, Karakiewicz PI, Palapattu GS, et al. Outcomes of radical cystectomy for transitional cell carcinoma of the bladder: a contemporary series from the Bladder Cancer Research Consortium. J Urol 2006;176:2414-22; discussion 22.

11. Sanchez-Ortiz RF, Huang WC, Mick R, et al. An interval longer than 12 weeks between the diagnosis of muscle invasion and cystectomy is associated with worse outcome in bladder carcinoma. J Urol 2003;169:110-5; discussion 5.

12. Mahmud SM, Fong B, Fahmy N, et al. Effect of preoperative delay on survival in patients with bladder cancer undergoing cystectomy in Quebec: a population based study. J Urol 2006; $175: 78-83$.

13. Fradet $Y$, Aprikian A, Dranitsaris $G$, et al. Does prolonging the time to bladder cancer surgery affect long-term cancer control: a systematic review of the literature. Can I Urol 2006;13(Suppl 3):37-47.

14. Lee CT, Madii R, Daignault $S$, et al. Cystectomy delay more than 3 months from initial bladder cancer diagnosis results in decreased disease specific and overall survival. J Urol 2006;175:1262-7.

15. Skinner DG, Daniels JR, Russell CA, et al. The role of adjuvant chemotherapy following cystectomy for invasive bladder cancer: a prospective comparative trial. J Urol 1991;145:459-64; discussion 64-7.

16. Stockle M, Meyenburg W, Wellek S, et al. Advanced bladder cancer (stages pT3b, pT4a, pN1 and pN2): improved sunvival after radical cystectomy and 3 adjuvant cycles of chemotherapy. Results of a controlled prospective study. J Urol 1992; 148:302-6; discussion 6-7.

17. Stockle M, Meyenburg W, Wellek $S$, et al. Adjuvant polychemotherapy of nonorganconfined bladder cancer after radical cystectomy revisited: long-term results of a controlled prospective study and further clinical experience. J Urol 1995;153:47-52.

18. Lehmann J, Franzaring L, Thuroff J, et al. Complete long-term survival data from a trial of adjuvant chemotherapy vs control after radical cystectomy for locally advanced bladder cancer. BJU Int 2006;97:42-7.

19. Freiha F, Reese J, Torti FM. A randomized trial of radical cystectomy versus radical cystectomy plus cisplatin, vinblastine and methotrexate chemotherapy for muscle invasive bladder cancer. J Urol 1996;155:495-9; discussion 499-500.

20. Adjuvant chemotherapy in invasive bladder cancer: a systematic review and meta-analysis of individual patient data Advanced Bladder Cancer (ABC) Meta-analysis Collaboration. Eur Urol 2005;48:189-99; discussion 199-201.

21. Studer UE, Bacchi M, Biedermann C, et al. Adjuvant cisplatin chemotherapy following cystectomy for bladder cancer: results of a prospective randomized trial. J Urol 1994;152:81-4.

Correspondence: Dr. Alan So, the Prostate Centre, Vancouver General Hospital, 2660 0ak St., Vancouver BC V6H 3Z6; alan.so@ubc.ca 